\title{
On geodesic homotopies of controlled width and conjugacies in isometry groups
}

\author{
Gerasim Kokarev
}

\begin{abstract}
We give an analytical proof of the Poincaré-type inequalities for widths of geodesic homotopies between equivariant maps valued in Hadamard metric spaces. As an application we obtain a linear bound for the length of an element conjugating two finite lists in a group acting on an Hadamard space.
\end{abstract}

Mathematics Subject Classification (2010). 53C23, 58E20, 20F65.

Keywords. Homotopy width, harmonic maps, Hadamard space, decision problems.

\section{Introduction}

Let $M$ and $M^{\prime}$ be smooth Riemannian manifolds without boundary. For a smooth mapping $u: M \rightarrow M^{\prime}$ by $E(u)$ we denote its energy

$$
E(u)=\int_{M}\|d u(x)\|^{2} d \operatorname{vol}(x),
$$

where the norm of the linear operator $d u(x): T_{x} M \rightarrow T_{u(x)} M^{\prime}$ is induced by the Riemannian metrics on $M$ and $M^{\prime}$. Let $u$ and $v$ be smooth homotopic mappings of $M$ to $M^{\prime}$ and $H(s, \cdot)$ be a smooth homotopy between them. The $L_{2}$-width $W_{2}(H)$ of $H$ is defined as the $L_{2}$-norm of the function

$$
\ell_{H}(x)=\text { the length of the curve } s \mapsto H(s, x), \quad x \in M \text {. }
$$

A smooth homotopy $H(s, x)$ is called geodesic if for each $x \in M$ the track curve $s \mapsto H(s, x)$ is a geodesic.

In [7], [8] Kappeler, Kuksin, and Schroeder prove the following geometric inequality for the $L_{2}$-widths of geodesic homotopies when the target manifold $M^{\prime}$ is non-positively curved.

Width Inequality I. Let $M$ and $M^{\prime}$ be compact Riemannian manifolds and suppose that $M^{\prime}$ has non-positive sectional curvature. Let $\zeta$ be a homotopy class of maps 
of $M$ to $M^{\prime}$. Then there exist constants $C_{*}$ and $C$ with the following property: any smooth homotopic maps $u$ and $v \in \zeta$ can be joined by a geodesic homotopy $H$ whose $L_{2}$-width $W_{2}(H)$ is controlled by the energies of $u$ and $v$,

$$
W_{2}(H) \leqslant C_{*}\left(E^{1 / 2}(u)+E^{1 / 2}(v)\right)+C .
$$

Moreover, if the sectional curvature of $M^{\prime}$ is strictly negative, the constants $C_{*}$ and $C$ can be chosen to be independent of a homotopy class $\zeta$.

This inequality can be viewed as a version of the Poincaré inequality for mappings between manifolds. It also has an isoperimetric flavour; it says that the 'measure' of the cylinder induced by the homotopy is estimated in terms of the 'measure' of its boundary. Inequality (1.2) is a key ingredient in the proof of compactness results for perturbed harmonic map equation [7], [9]. The latter, combined with old results of Uhlenbeck, yields Morse inequalities for harmonic maps with potential [10].

The proof of Width Inequality I in [7], [8] is based on an analogous inequality for maps of metric graphs; see [7], Th. 5.1. In more detail, let $G$ be a finite graph and $u: G \rightarrow M^{\prime}$ be a smooth map, that is, whose restriction to every edge is smooth. The length $L(u)$ of $u$ is defined as the sum of the lengths of the images of the edges. By the $L_{\infty}$-width $W_{\infty}(H)$ of a homotopy $H$ we mean the $L_{\infty}$-norm of the length function $\ell_{H}(x)$, given by (1.1).

Width Inequality II. Let $G$ be a finite graph and $M^{\prime}$ be a compact manifold of non-positive sectional curvature. Let $\zeta$ be a homotopy class of maps $G \rightarrow M^{\prime}$. Then there exist constants $C_{*}$ and $C$ with the following property: any smooth homotopic maps $u$ and $v \in \zeta$ can be joined by a geodesic homotopy $H$ such that

$$
W_{\infty}(H) \leqslant C_{*}(L(u)+L(v))+C .
$$

Moreover, if the sectional curvature of $M^{\prime}$ is strictly negative, the constants $C_{*}$ and $C$ can be chosen to be independent of a homotopy class $\zeta$.

The purpose of this note is twofold: firstly, we generalise the width inequalities to the framework of equivariant maps, which we assume to be valued in Hadamard metric spaces. This, in particular, includes width inequalities for homotopies between maps into non-compact target spaces, completing the previous results [8] in this direction. In contrast with the geometric methods in [7], [8] (and also algebraic in [2]), we give an analytical proof of the width inequalities via harmonic map theory.

Secondly, we use width inequalities for equivariant maps of trees to study the multiple conjugacy problem for finitely generated groups $\Lambda$ acting by isometries on Hadamard spaces. More precisely, under some extra hypotheses, we obtain a linear estimate for the conjugator length in these groups: given two finite conjugate lists of elements $\left(a_{i}\right)_{1 \leqslant i \leqslant N}$ and $\left(b_{i}\right)_{1 \leqslant i \leqslant N}$ in $\Lambda$ there exists a conjugator $g \in \Lambda$, 
$b_{i}=g^{-1} a_{i} g$ such that

$$
|g| \leqslant C_{*} \sum_{i=1}^{N}\left(\left|a_{i}\right|+\left|b_{i}\right|\right)+C,
$$

where $|\cdot|$ stands for the length $d(\cdot, e)$ in the word metric on $\Lambda$. If the group $\Lambda$ has a soluble word problem, then the latter estimate yields immediately the solubility of the multiple conjugacy problem in $\Lambda$.

Acknowledgments. The author is grateful to Sergei Kuksin and Dieter Kotschick for their interest in the work and a number of discussions on the subject. The paper was written when the author held a personal EPSRC fellowship at the University of Edinburgh.

\section{Statements and discussion of results}

2.1. Width inequalities for equivariant maps. Let $M$ be a compact Riemannian manifold without boundary; we denote by $\widetilde{M}$ its universal cover and by $\Gamma$ the fundamental group $\pi_{1}(M)$. Let $(Y, d)$ be an Hadamard space; that is a complete length space of non-positive curvature in the sense of Alexandrov (see Section 3 for a precise definition). Denote by $\rho$ a representation of $\Gamma$ in the isometry group of $Y$. Recall that a map $u: \tilde{M} \rightarrow Y$ is called $\rho$-equivariant if

$$
u(g \cdot x)=\rho(g) \cdot u(x) \text { for all } x \in \tilde{M}, g \in \Gamma .
$$

For $\rho$-equivariant maps $u$ and $v$ the real-valued functions $d(u(x), v(x))$, where $x \in$ $\tilde{M}$, are invariant with respect to the domain action and, hence, are defined on the quotient $M=\tilde{M} / \Gamma$. In particular, the quantity

$$
d_{2}(u, v)=\left(\int_{M} d^{2}(u(x), v(x)) d \operatorname{vol}(x)\right)^{1 / 2}
$$

defines a metric on the space of locally $L_{2}$-integrable $\rho$-equivariant maps. The latter can be also regarded as the $L_{2}$-width of a unique geodesic homotopy between $\rho$ equivariant maps. If $u$ is a locally Sobolev $W^{1,2}$-smooth $\rho$-equivariant map, then its energy density measure $|d u|^{2} d$ vol (see Section 3 ) is also $\Gamma$-invariant and the energy of $u$ is defined as the integral

$$
E(u)=\int_{M}|d u|^{2} d \text { vol } .
$$

Recall that the ideal boundary of $Y$ is defined as the set of equivalence classes of asymptotic geodesic rays; two rays are asymptotic if they remain at a bounded distance from each other. Clearly, any action of $\Gamma$ by isometries on $Y$ extends to the action on the ideal boundary. 
Theorem 1. Let $M$ be a compact Riemannian manifold without boundary and $Y$ be a locally compact Hadamard space. Let $\Gamma$ be the fundamental group of $M$ and $\rho: \Gamma \rightarrow \operatorname{Isom}(Y)$ be its representation whose image does not fix a point on the ideal boundary of $Y$. Then there exists a constant $C_{*}$ such that for any $\rho$-equivariant locally $W^{1,2}$-smooth maps $u$ and $v$ the $L_{2}$-width of a geodesic homotopy $H$ between them satisfies the inequality

$$
W_{2}(H) \leqslant C_{*}\left(E^{1 / 2}(u)+E^{1 / 2}(v)\right) .
$$

The proof appears in Section 4. The idea is to prove first a similar inequality when one of the maps is an energy minimiser, and then to use compactness properties of the moduli space formed by such minimisers. The former is based on a compactness argument, mimicking the proof of the classical Poincaré inequality.

Below we state a version of Theorem 1 for equivariant maps of trees. First, we introduce more notation. Let $G$ be a finite connected graph without terminals and $\Gamma$ be its fundamental group $\pi_{1}(G)$. By $T$ we denote the universal covering tree of $G$; the group $\Gamma$ acts naturally on $T$ by the deck transformations. As above the symbol $\rho$ denotes a representation of $\Gamma$ in the isometry group of an Hadamard space $Y$. For a locally rectifiable $\rho$-equivariant map $u: T \rightarrow Y$, its length density measure $|d u| d t$ (see Section 3) is $\Gamma$-invariant and the length of $u$ is defined as the integral

$$
L(u)=\int_{G}|d u| d t .
$$

Theorem 2. Let $G$ be a finite graph and $Y$ be a locally compact Hadamard space. Let $\Gamma$ be the fundamental group of $G$ and $\rho: \Gamma \rightarrow \operatorname{Isom}(Y)$ be its representation whose image does not fix a point on the ideal boundary of $Y$. Then there exists a constant $C_{*}$ such that for any locally rectifiable $\rho$-equivariant maps $u$ and $v$ the $L_{\infty}$-width of a geodesic homotopy between them satisfies the inequality

$$
W_{\infty}(H) \leqslant C_{*}(L(u)+L(v)) .
$$

Example. Let $M^{\prime}$ be a non-compact Riemannian manifold whose sectional curvature is negative and bounded away from zero and the injectivity radius is positive. Let $\rho: \Gamma \rightarrow \pi_{1}\left(M^{\prime}\right)$ be a homomorphism whose image is neither trivial nor infinite cyclic. Then the latter does not fix a point on the ideal boundary of the universal cover of $M^{\prime}$. Indeed, the group $\rho(\Gamma)$ is generated by hyperbolic elements (regarded as isometries of the universal cover), see [8], Lemma B.1, and the statement follows from the results in [4], Sect. 6. Thus, as a particular case, Theorem 2 contains the width inequality for homotopies between maps from $G$ to $M^{\prime}$; this is the situation considered in [8], Th. 0.1. Under the same hypothesis on $M^{\prime}$, Theorem 1 yields an analogous $L_{2}$-width inequality for geodesic homotopies between maps from domains whose dimension is greater than one. The latter statement does not seem to be achievable via the methods in [7], [8]. 
We proceed with width inequalities for representations in co-compact subgroups of $\operatorname{Isom}(Y)$. Recall that an action of a group $\Lambda$ on a metric space $(Y, d)$ is said to be $c o$ compact if the quotient $Y / \Lambda$ is compact. Further, the action of $\Lambda$ is said to be proper if for each $y \in Y$ there exists $r>0$ such that the set $\{g \in \Lambda \mid g \cdot B(y, r) \cap B(y, r) \neq \varnothing\}$ is finite. For a homomorphism $\rho: \Gamma \rightarrow \Lambda$ we denote below by $Z$ the centraliser of the image $\rho(\Gamma)$ in $\Lambda$.

Theorem 3. Let $M$ be a compact Riemannian manifold without boundary and $Y$ be a locally compact Hadamard space. Let $\Lambda$ be a group acting properly and cocompactly by isometries on $Y$. Denote by $\Gamma$ the fundamental group of $M$ and let $\rho$ be a homomorphism $\Gamma \rightarrow \Lambda$. Then there are constants $C_{*}$ and $C$ such that for any $\rho$-equivariant locally $W^{1,2}$-smooth maps $u$ and $v$ there exists an element $h \in Z$ such that the $L_{2}$-width of a geodesic homotopy $H$ between $u$ and $h \cdot v$ satisfies the inequality

$$
W_{2}(H) \leqslant C_{*}\left(E^{1 / 2}(u)+E^{1 / 2}(v)\right)+C .
$$

Theorem 4. Let $G$ be a finite graph and $Y$ be a locally compact Hadamard space. Let $\Lambda$ be a group acting properly and co-compactly by isometries on $Y$. Denote by $\Gamma$ the fundamental group of $G$ and let $\rho$ be a homomorphism $\Gamma \rightarrow \Lambda$. Then there are constants $C_{*}$ and $C$ such that for any locally rectifiable $\rho$-equivariant maps $u$ and $v$ there exists an element $h \in Z$ such that the $L_{\infty}$-width of a geodesic homotopy $H$ between $u$ and $h \cdot v$ satisfies the inequality

$$
W_{\infty}(H) \leqslant C_{*}(L(u)+L(v))+C .
$$

Remark. If the homomorphism $\rho: \Gamma \rightarrow \Lambda$ in the theorems is trivial, then the second constant $C$ is equal to $\operatorname{diam}(Y / \Lambda) \operatorname{vol}^{1 / 2} M$ and $\operatorname{diam}(Y / \Lambda)$ in the $L_{2^{-}}$and $L_{\infty^{-}}$ versions respectively. For non-trivial representations of $\Gamma$ it can be chosen to be zero.

Example. As a partial case, when the action of $\Lambda$ is free, Theorems 3 and 4 above contain width inequalities for homotopies between continuous $W^{1,2}$-smooth maps valued in a compact metric space $Y / \Lambda$. The choice of an element $h \in Z$ in this setting corresponds to the choice of the homotopy between maps. Indeed, recall that the fundamental group of the space formed by continuous maps homotopic to $u: M \rightarrow Y / \Lambda$ is equal to the centraliser of the image $u_{*}\left(\pi_{1}(M)\right)$ in $\Lambda$.

2.2. Conjugacies of finite lists in isometry groups. Now we describe some applications of the width inequalities to geometric group theory. First, recall that a discrete subgroup $\Lambda$ in a Lie group $\boldsymbol{G}$ is called the lattice if the quotient $\boldsymbol{G} / \Lambda$ carries a finite $\boldsymbol{G}$-invariant measure. Such a lattice is always finitely generated provided the group $\boldsymbol{G}$ is semi-simple and has rank $\geqslant 2$; see ref. in [12]. Choose a finite system of generators $\left(g_{i}\right)$ of $\Lambda$ and consider the word metric $d(\cdot, \cdot)$ on $\Lambda$ associated with 
the Cayley graph determined by the generators. By $|g|$ we denote below the length $d(g, e)$, the distance between an element $g$ and the neutral element $e$.

The following statements are essentially consequences of Theorems 2 and 4 and are explained in Section 5.

Theorem 5. Let $\boldsymbol{G}$ be a semi-simple Lie group of rank $\geqslant 2$ all of whose simple factors are non-compact. Let $\Lambda$ be an irreducible lattice in $\boldsymbol{G}$ and $\left(a_{i}\right)_{1 \leqslant i \leqslant N}$ be a finite list of elements in $\Lambda$ which does not fix a point on the ideal boundary of the associated symmetric space. Then for any conjugate (in $\Lambda$ ) list $\left(b_{i}\right)_{1 \leqslant i \leqslant N}$ any conjugating element $g \in \Lambda, b_{i}=g^{-1} a_{i} g$ satisfies the inequality

$$
|g| \leqslant C_{*} \sum_{i=1}^{N}\left(\left|a_{i}\right|+\left|b_{i}\right|\right)+C,
$$

where the constants depend only on the conjugacy class of the lists. In particular, for such two given lists the set of conjugating elements is finite.

Remark. An analogous statement holds if $\Lambda$ is an irreducible lattice in an almost simple $p$-adic algebraic Lie group of rank $\geqslant 2$. In this case we consider lists which do not fix points on the ideal boundary of the associated Euclidean building.

Example. When the group $\boldsymbol{G}$ is algebraic, the hypothesis on the finite list $\left(a_{i}\right)$ is satisfied if, for example, the elements $a_{i}$ 's generate a lattice (e.g., the whole group $\Lambda$ ) in $\boldsymbol{G}$. Indeed, by Borel's density theorem the latter is Zariski dense in $\boldsymbol{G}$ and, hence, does not fix a point on the ideal boundary of the associated symmetric space.

The estimate above yields immediately an algorithm deciding whether a given list of elements in $\Lambda$ is conjugate to the list $\left(a_{i}\right)$ in the theorem. This is a special case of the more general result due to Grunewald and Segal [6]: the conjugacy problem for finite lists in arithmetic groups is soluble. (Any irreducible lattice in a semi-simple Lie group of rank $\geqslant 2$ is arithmetic by Margulis' theorem.) However, we do not know whether the linear estimate for the length of the conjugating element holds under weaker hypotheses than in Theorem 5.

We proceed with the conjugacy problem for finite lists in groups which act properly and co-compactly on Hadamard spaces by isometries. Recall that such groups are necessarily finitely presented; see [1], I.8.11. As above by $|g|$ we denote the length $d(g, e)$ in the word metric.

Theorem 6. Let $Y$ be a locally compact Hadamard space and $\Lambda$ be a group acting properly and co-compactly by isometries on $Y$. Then for any finite conjugate lists $\left(a_{i}\right)_{1 \leqslant i \leqslant N}$ and $\left(b_{i}\right)_{1 \leqslant i \leqslant N}$ of elements in $\Lambda$ there exists an element $g \in \Lambda$ with $b_{i}=g^{-1} a_{i} g$ such that

$$
|g| \leqslant C_{*} \sum_{i=1}^{N}\left(\left|a_{i}\right|+\left|b_{i}\right|\right)+C,
$$


where the constants depend only on the conjugacy class of the lists. Further, there exists an algorithm deciding whether two given finite lists of elements in $\Lambda$ are conjugate.

When the list $\left(a_{i}\right)$ in the theorem consists of a single element, the solubility of the conjugacy problem is well known. It is, for example, a consequence of an exponential bound for the length of the conjugating element in [1], III. Г.1.12. Theorem 6 shows that this exponential bound can be improved to a linear bound (with the cost that the constants become dependant on the conjugacy class), thus, giving a partial affirmative answer to Question 10.4 in [3]. In the context of decision problems it is worth noting that there are finitely presented groups in which the conjugacy problem for elements is soluble, but the conjugacy problem for finite lists is not. We refer to [2] for the explicit examples. Finally, mention that in [2] Bridson and Howie, using purely algebraic methods, prove a closely related linear estimate for the length of the conjugating (two finite lists) element in Gromov hyperbolic groups. An analogous conjugator length estimate is also known for pseudo-Anosov elements in the mapping class group, thus reflecting its hyperbolic character, see [13]. For a more general discussion on the geometry of the conjugacy problem, we refer to the survey talk by Tim Riley [15].

\section{Preliminaries}

3.1. Sobolev spaces of maps to metric targets. We recall some background material on Sobolev spaces of maps valued in a metric space. The details can be found in [11].

Let $\Omega$ be a Riemannian domain and $(Y, d)$ be an arbitrary metric space. We suppose that $\Omega$ is endowed with a Lebesgue measure $d$ vol induced by the Riemannian volume. A measurable map $u: \Omega \rightarrow Y$ is called locally $L_{2}$-integrable if it has a separable essential range and for which $d(u(\cdot), Q)$ is a locally $L_{2}$-integrable function on $\Omega$ for some $Q \in Y$ (and, hence, by the triangle inequality for any $Q \in Y$ ). If the domain $\Omega$ is bounded, then the function

$$
d_{2}(u, v)=\left(\int_{\Omega} d^{2}(u(x), v(x)) d \operatorname{vol}(x)\right)^{1 / 2}
$$

defines a metric on the space of locally $L_{2}$-integrable maps. The latter is complete provided $Y$ is complete.

The approximate energy density of a locally $L_{2}$-integrable map $u$ is defined for $\varepsilon>0$ by

$$
e_{\varepsilon}(u)(x)=\int_{S_{\varepsilon}(x)} \frac{d^{2}\left(u(x), u\left(x^{\prime}\right)\right)}{\varepsilon^{n+1}} d \operatorname{vol}\left(x^{\prime}\right),
$$

where $S_{\varepsilon}(x)$ denotes the $\varepsilon$-sphere centred at $x$ and $n$ stands for the dimension of $\Omega$. The function $e_{\varepsilon}(x)$ is non-negative and locally $L_{1}$-integrable. 
Definition. The energy $E(u)$ of a locally $L_{2}$-integrable map $u$ is defined by

$$
E(u)=\sup _{0 \leqslant f \leqslant 1}\left(\lim _{\varepsilon \rightarrow 0} \sup \int_{\Omega} f e_{\varepsilon}(u) d \text { vol }\right),
$$

where the sup is taken with respect to compactly supported continuous functions which take values between 0 and 1 . A locally $L_{2}$-integrable map $u$ is called locally $W^{1,2}$-smooth if for any relatively compact domain $D \subset \Omega$ the energy $E\left(\left.u\right|_{D}\right)$ is finite.

Due to the results of Korevaar and Schoen [11], Sect. 1, a locally $L_{2}$-integrable map $u$ is locally $W^{1,2}$-smooth if and only if there exists a locally $L_{1}$-integrable function $e(u)$ such that the measures $e_{\varepsilon}(u) d$ vol converge weakly to the measure $e(u) d$ vol as $\varepsilon \rightarrow 0$. The function $e(u)$, also denoted by $|d u|^{2}$, is called the energy density of $u$, and the energy $E(u)$ is equal to the total mass $\int e(u) d$ vol.

Now suppose that the domain $\Omega$ is 1-dimensional, that is an interval $I=(a, b)$. For a map $u: I \rightarrow Y$ one can also define the approximate length density by

$$
l_{\varepsilon}(u)(t)=\frac{d(u(t), u(t+\varepsilon))+d(u(t), u(t-\varepsilon))}{\varepsilon}, \quad t \in I .
$$

Then the length of $u$ is defined by the formula similar to that for the energy,

$$
L(u)=\sup _{0 \leqslant f \leqslant 1}\left(\lim _{\varepsilon \rightarrow 0} \sup \int_{I} f l_{\varepsilon}(u) d t\right),
$$

where the sup is taken with respect to compactly supported continuous functions. A map $u: I \rightarrow Y$ is called rectifiable if its length is finite. In this case there exists a length density function (or speed function) $l(u)$ such that the length $L(u)$ equals $\int l(u) d t$.

3.2. Hadamard spaces. Recall that an Hadamard space $(Y, d)$ is a complete metric space which satisfies the following two hypotheses:

(i) Length space. For any two points $y_{0}$ and $y_{1} \in Y$ there exists a rectifiable curve $\gamma$ from $y_{0}$ to $y_{1}$ such that

$$
d\left(y_{0}, y_{1}\right)=\text { length }(\gamma)
$$

We call such a curve $\gamma$ geodesic.

(ii) Triangle comparison. For any three points $P, Q$, and $R$ in $Y$ and the choices of geodesics $\gamma_{P Q}, \gamma_{Q R}$, and $\gamma_{R P}$ connecting the respecting points denote by $\bar{P}$, $\bar{Q}$, and $\bar{R}$ the vertices of the (possibly degenerate) Euclidean triangle with the side lengths $\ell\left(\gamma_{P Q}\right), \ell\left(\gamma_{Q R}\right)$, and $\ell\left(\gamma_{R P}\right)$ respectively. Let $Q_{\lambda}$ be a point on the geodesic $\gamma_{Q R}$ which is a fraction $\lambda, 0 \leqslant \lambda \leqslant 1$, of the distance from $Q$ to $R$;

$$
d\left(Q_{\lambda}, Q\right)=\lambda d(Q, R), \quad d\left(Q_{\lambda}, R\right)=(1-\lambda) d(Q, R) .
$$


Denote by $\bar{Q}_{\lambda}$ an analogous point on the side $\bar{Q} \bar{R}$ of the Euclidean triangle. The triangle comparison hypothesis says that the metric distance $d\left(P, Q_{\lambda}\right)$ (from $Q_{\lambda}$ to the opposite vertex) is bounded above by the Euclidean distance $\left|\bar{P}-\bar{Q}_{\lambda}\right|$. This inequality can be written in the form

$$
d_{P Q_{\lambda}}^{2} \leqslant(1-\lambda) d_{P Q}^{2}+\lambda d_{P R}^{2}-\lambda(1-\lambda) d_{Q R}^{2}
$$

It is a direct consequence of the property (ii) above that geodesics in an Hadamard space are unique. It is also a consequence of geodesic uniqueness that an Hadamard space has to be simply-connected [1], II.1. Examples include symmetric spaces of non-compact type and Euclidean buildings, simply-connected manifolds of nonpositive sectional curvature, Hilbert spaces, simply-connected Euclidean or hyperbolic simplicial complexes satisfying certain local link conditions [1], II.5.4. Another class of examples is provided by the following proposition.

Proposition 1. Let $M$ be a compact Riemannian manifold without boundary and $(Y, d)$ be an Hadamard space. Let $\rho$ be a representation of the fundamental group $\Gamma=\pi_{1}(M)$ in the group of isometries of $Y$. Then the space of $\rho$-equivariant locally $L_{2}$-integrable maps from $\tilde{M}$ to $Y$ endowed with the metric (2.1) is an Hadamard space.

The proof follows straightforward from the definitions: the geodesics in the new space are geodesic homotopies and the triangle comparison hypothesis follows by integration of relation (3.1).

A useful consequence of the triangle comparison hypothesis is the following quadrilateral comparison property due to Reshetnyak [14] (we refer to [11], Cor. 2.1.3, for a proof).

Proposition 2. Let $(Y, d)$ be an Hadamard space and $P, Q, R$, and $S$ be an ordered sequence of points in $Y$. For $0 \leqslant \lambda, \mu \leqslant 1$ define $P_{\lambda}$ to be the point which is the fraction $\lambda$ of the way from $P$ to $S$ (on the geodesic $\gamma_{P S}$ ) and $Q_{\mu}$ to be the point which is the fraction $\mu$ of the way from $Q$ to $R$ (on the opposite geodesic $\gamma_{Q R}$ ). Then for any $0 \leqslant \alpha, t \leqslant 1$ the following inequality holds:

$d_{P_{t} Q_{t}}^{2} \leqslant(1-t) d_{P Q}^{2}+t d_{R S}^{2}-t(1-t)\left[\alpha\left(d_{P S}-d_{Q R}\right)^{2}+(1-\alpha)\left(d_{R S}-d_{P Q}\right)^{2}\right]$.

Setting $\alpha$ to be equal to zero in this inequality, we deduce the convexity of the distance between geodesics

$$
d_{P_{t} Q_{t}} \leqslant(1-t) d_{P Q}+t d_{R S}
$$

This implies the following energy convexity property. Let $u$ and $v$ be locally $W^{1,2}$ smooth maps from the Riemannian domain $\Omega$ to an Hadamard space $(Y, d)$. Let $H(s, \cdot)$ be a geodesic homotopy between $u$ and $v$; the point $H(s, x)$ is the fraction $s$ 
of the way from $u(x)$ to $v(x)$, where $x \in \Omega$. Then for any $s$ the map $H(s, \cdot)$ is locally $W^{1,2}$-smooth and for any relatively compact domain $D \subset \Omega$ its energy satisfies the inequality

$$
E^{1 / 2}\left(H_{s}\right) \leqslant(1-s) E^{1 / 2}(u)+s E^{1 / 2}(v) .
$$

Inequality (3.2) also yields the length convexity along geodesic homotopies. More precisely, let $u$ and $v$ be rectifiable paths in $(Y, d)$ and let $H(s, \cdot)$ be a geodesic homotopy between the parameterised by the arc length as above. Then for any $s$ the map $H(s, \cdot)$ is rectifiable and its length satisfies the inequality

$$
L\left(H_{s}\right) \leqslant(1-s) L(u)+s L(v) .
$$

Another consequence of the triangle comparison hypothesis is the existence of the nearest point projection $\pi: Y \rightarrow A$ onto a convex subset $A$. In more detail, if $(Y, d)$ is an Hadamard space and $A$ is its non-empty closed convex subset, then for any $y \in Y$ there exists a unique point $a \in A$ which minimises the distance $d(y, a)$ among all points in $A$; see [11], Prop. 2.5.4.

3.3. Some properties of harmonic maps. Let $M$ be a compact Riemannian manifold without boundary and $(Y, d)$ be an Hadamard space. As above by $\Gamma$ we denote the fundamental group of $M$ and by $\rho: \Gamma \rightarrow \operatorname{Isom}(Y)$ its representation in the isometry group of $Y$. We consider $\rho$-equivariant locally $W^{1,2}$-smooth maps $u$ from the universal cover $\tilde{M}$ to $Y$. The energy density of such a map $u$ is a $\Gamma$-invariant function on $\tilde{M}$, which can be also regarded as a function on the quotient $M=\widetilde{M} / \Gamma$. In particular, by the energy $E(u)$ we understand the integral $\int_{M} e(u) d$ vol. We call a $\rho$ equivariant map harmonic if it minimises the energy among all $\rho$-equivariant locally $W^{1,2}$-smooth maps.

The following statement is a straightforward consequence of the energy convexity, formula (3.3). We state it as a proposition for the convenience of references.

Proposition 3. Under the hypotheses above, let $u$ and $v$ be two $\rho$-equivariant harmonic maps and $H(s, \cdot)$ be a geodesic homotopy between them; the point $H(s, x)$ is the fraction s of the way from $u(x)$ and $v(x)$, where $x \in \tilde{M}$. Then for each $s$ the map $H(s, \cdot)$ is also $\rho$-equivariant harmonic and the energy $E\left(H_{s}\right)$ does not depend on $s$.

We proceed with the Lipschitz continuity of harmonic maps. The following proposition is a consequence of the result by Korevaar and Schoen [11], [Th. 2.4.6].

Proposition 4. Under the hypotheses above, any $\rho$-equivariant harmonic map $u$ is Lipschitz continuous and its Lipschitz constant is bounded above by $C \cdot E^{1 / 2}(u)$, where the constant $C$ depends on the manifold $M$ and its metric only.

Now let $G$ be a finite connected graph without terminals and $\Gamma$ be its fundamental group. By $T$ we denote the universal covering tree of $G$. Similarly to the discussion 
above, for a locally rectifiable $\rho$-equivariant map $u: T \rightarrow Y$ the length density function $l(u)$ is $\Gamma$-invariant and, hence, descends to the quotient $G=T / \Gamma$. In particular, by the length $L(u)$ we understand the integral $\int_{G} l(u) d t$. It is straightforward to see that if a map $u$ minimises the length among all locally rectifiable $\rho$-equivariant maps, then its restriction to every edge is a geodesic. If the latter has a constant-speed parameterisation on every edge, then it is also harmonic and the length of every edge $u_{I}$ satisfies the relation $L^{2}(u)=E\left(u_{I}\right)(b-a)$, see [5], Lemma 12.5. Conversely, if $u$ is a $\rho$-equivariant harmonic map, then its restriction to every edge is a constant-speed geodesic whose squared length is proportional to the energy as above. In particular, the length is constant on the set of $\rho$-equivariant harmonic maps, where it achieves its minimum.

\section{Proofs of the width inequalities}

We start with the following lemma.

Main Lemma I. Let $M$ be a compact Riemannian manifold without boundary and $(Y, d)$ be a locally compact Hadamard space. Let $\rho: \Gamma \rightarrow \operatorname{Isom}(Y)$ be a representation of the fundamental group $\Gamma=\pi_{1}(M)$. Suppose that the moduli space Harm, formed by $\rho$-equivariant harmonic maps $\tilde{M} \rightarrow Y$, is non-empty and bounded in the $L_{2}$-metric. Then there exists a positive constant $C_{*}$ with the following property: for any $\rho$-equivariant locally $W^{1,2}$-smooth map $u$ there exists a harmonic map $\bar{u} \in$ Harm such that

$$
d_{2}(u, \bar{u}) \leqslant C_{*}\left(E^{1 / 2}(u)-E_{*}^{1 / 2}\right),
$$

where $E_{*}=E(\bar{u})$ is the energy minimum among $\rho$-equivariant maps.

Proof. First, note that inequality (4.1) is invariant under the rescaling of the metric on the target space $Y$. Hence, it is sufficient to prove the lemma under the assumption that

$$
\text { the distance } d_{2}(u, \bar{u}) \text { is not less than one. }
$$

Suppose the contrary. Then there exists a sequence of maps $u_{k}$ such that

$$
d_{2}\left(u_{k}, \bar{u}\right) \geqslant k\left(E^{1 / 2}\left(u_{k}\right)-E^{1 / 2}(\bar{u})\right)
$$

for any $\bar{u} \in$ Harm. For each $u_{k}$ choose a harmonic map $\bar{u}_{k}$ at which the infimum

$$
d_{2}(u, \bar{u})=\inf \left\{d_{2}(u, v) \mid v \in \operatorname{Harm}\right\}
$$

is attained. Such a harmonic map $\bar{u}$ clearly exists: it is the value of $u$ under the nearest point projection onto Harm. (The lower semicontinuity of the energy [11], Th. 1.6.1, and Proposition 3 imply that Harm is a closed convex subset in the Hadamard space of $\rho$-equivariant locally $L_{2}$-integrable maps.) 
Denote by $H_{s}^{k}$, where $s \in[0,1]$, a geodesic homotopy between $\bar{u}_{k}$ and $u_{k}$; we set $H_{0}^{k}=\bar{u}_{k}$ and $H_{1}^{k}=u_{k}$. Assuming that the parameter $s$ is proportional to the arc length, we obtain

$$
d_{2}\left(H_{s}^{k}, H_{0}^{k}\right)=s \cdot d_{2}\left(u_{k}, \bar{u}_{k}\right) \geqslant s \cdot k\left(E^{1 / 2}\left(u_{k}\right)-E^{1 / 2}\left(\bar{u}_{k}\right)\right) .
$$

Recall the energy $E^{1 / 2}(\cdot)$ is convex along geodesic homotopies;

$$
s\left(E^{1 / 2}\left(u_{k}\right)-E^{1 / 2}\left(\bar{u}_{k}\right)\right) \geqslant E^{1 / 2}\left(H_{s}^{k}\right)-E^{1 / 2}\left(H_{0}^{k}\right) .
$$

Combining the last two inequalities we conclude that

$$
d_{2}\left(H_{s}^{k}, H_{0}^{k}\right) \geqslant k\left(E^{1 / 2}\left(H_{s}^{k}\right)-E^{1 / 2}\left(H_{0}^{k}\right)\right) .
$$

Now choose a sequence of $s_{k} \in[0,1]$ such that the distance $d_{2}\left(H_{s_{k}}^{k}, H_{0}^{k}\right)$ equals one; by the assumption (4.2) this is possible. Then relation (4.3) implies that the sequence $E\left(H_{s_{k}}^{k}\right)$ converges to $E_{*}$ as $k \rightarrow+\infty$. Since the moduli space Harm is bounded in $L_{2}$-metric, the latter together with the choice of the $s_{k}$ 's implies that the sequence $H_{s_{k}}^{k}$ is bounded in the $W^{1,2}$-sense; that is

$$
d_{2}\left(H_{s_{k}}^{k}, w\right)+E\left(H_{s_{k}}^{k}\right) \leqslant C,
$$

where $w$ is a fixed $\rho$-equivariant map. Now by the version of Rellich's embedding theorem [11], Th. 1.13, we can find a subsequence $H_{s_{k}}^{k}$ (denoted by the same symbol) which converges in $L_{2}$-metric and point-wise to a locally $W^{1,2}$-smooth map $\bar{v}$. By the lower semi-continuity of the energy [11], Th. 1.6.1, the map $\bar{v}$ is energy minimising and by the point-wise convergence is $\rho$-equivariant. By the choice of the $s_{k}$ 's we clearly have

$$
d_{2}\left(H_{1}^{k}, H_{s_{k}}^{k}\right)=d_{2}\left(H_{1}^{k}, H_{0}^{k}\right)-d_{2}\left(H_{s_{k}}^{k}, H_{0}^{k}\right)=d_{2}\left(u_{k}, \bar{u}_{k}\right)-1 .
$$

Thus, the $L_{2}$-distance between the maps $u_{k}$ and $v$ can be estimated by

$$
d_{2}\left(u_{k}, \bar{v}\right) \leqslant d_{2}\left(H_{1}^{k}, H_{s_{k}}^{k}\right)+d_{2}\left(H_{s_{k}}^{k}, \bar{v}\right)=d_{2}\left(u_{k}, \bar{u}_{k}\right)+\left(d_{2}\left(H_{s_{k}}^{k}, \bar{v}\right)-1\right) .
$$

For sufficiently large $k$ the second term on the right-hand side is negative, and we arrive at a contradiction with the choice of the harmonic maps $\bar{u}_{k}$ 's.

The following lemma summarises known results (essentially due to [11]) on the moduli space Harm, formed by $\rho$-equivariant maps.

Lemma 1. Let $M$ be a compact Riemannian manifold without boundary and $Y$ be a locally compact Hadamard space. Let $\Gamma$ be the fundamental group of $M$ and $\rho: \Gamma \rightarrow \operatorname{Isom}(Y)$ be its representation whose image does not fix a point on the ideal boundary of $Y$. Then the moduli space Harm, formed by $\rho$-equivariant harmonic maps, is non-empty and compact in $C^{0}$-topology. 
Since there is no direct reference for the statement on the compactness of Harm and to make our paper more self-contained, we give a proof now.

Proof of Lemma 1. First, we explain the existence of a $\rho$-equivariant harmonic map. By [11], Th. 2.6.4, there exists an energy minimising sequence $\left\{u_{i}\right\}$ of equivariant Lipschitz continuous maps, whose Lipschitz constants are uniformly bounded. Let $\Omega$ be a fundamental domain for the action of $\Gamma$ on the universal cover $\tilde{M}$. We claim that under the hypotheses of the theorem the ranges $u_{i}(\Omega)$ are contained in a bounded subset of $Y$. Indeed, suppose the contrary. Then there exists a point $x \in \Omega$ such that the sequence $\left\{u_{i}(x)\right\}$ is unbounded in $Y$, i.e.,

$$
d\left(u_{i}(x), Q\right) \rightarrow+\infty \quad \text { for some } Q \in Y .
$$

For any $g \in \Gamma$ consider the sequence $d\left(\rho(g) \cdot u_{i}(x), u_{i}(x)\right)$. By the equivariance of the $u_{i}$ 's and the uniform boundedness of their Lipschitz constants we have

$$
d\left(\rho(g) \cdot u_{i}(x), u_{i}(x)\right) \leqslant C d(g \cdot x, x),
$$

and hence the quantities on the left hand side remain bounded as $i \rightarrow+\infty$. By the convexity of the distance between geodesics, relation (3.2), we see that the (Hausdorff) distances between the geodesic segments $\overline{Q u_{i}(x)}$ and $\rho(g) \cdot \overline{Q u_{i}(x)}$ also remain bounded as $i \rightarrow+\infty$. Since $Y$ is locally compact, we can find a subsequence of $u_{i}$, denoted by the same symbol, such that the segments $\overline{Q u_{i}(x)}$ converge on compact subsets to a geodesic ray $\sigma$ with initial point at $Q$. Then the distance between $\sigma$ and $\rho(g) \cdot \sigma$ is also bounded for any $g \in \Gamma$. This shows that $\sigma$ represents a fixed point for the action of $\rho(\Gamma)$ and leads to a contradiction.

Now, since $Y$ is locally compact, the Arzela-Ascoli theorem applies and we can find a subsequence of $u_{i}$ converging in $C^{0}$-topology to an energy-minimising and, hence, harmonic map. Thus, the moduli space Harm is non-empty.

Finally, we explain the compactness of Harm. Let $u_{i}$ be a sequence of $\rho$ equivariant harmonic maps. By Proposition 3 their energies coincide, and by Proposition 4 the $u_{i}$ 's are uniformly Lipschitz continuous. The same argument as above shows that the ranges $u_{i}(\Omega)$ are contained in a bounded subset of $Y$. Again by the Arzela-Ascoli theorem there exists a converging subsequence. By the lower semicontinuity of the energy the limit map is energy minimising and, hence, harmonic. Thus, the moduli space Harm is compact in $C^{0}$-topology among $\rho$-equivariant harmonic maps.

Proof of Theorem 1. By Lemma 1, Main Lemma I applies: for given $\rho$-equivariant maps $u$ and $v$ we can find harmonic $\rho$-equivariant maps $\bar{u}$ and $\bar{v}$ such that $d_{2}(u, \bar{u})$ and $d_{2}(v, \bar{v})$ are estimated as in (4.1). By Lemma 1 the moduli space Harm is compact and, hence, the distance $d_{2}(\bar{u}, \bar{v})$ is uniformly bounded. The $L_{2}$-width of a geodesic homotopy $H$ between $u$ and $v$ is the distance $d_{2}(u, v)$, and by the triangle inequality we have

$$
W_{2}(H) \leqslant d_{2}(u, \bar{u})+d_{2}(\bar{u}, \bar{v})+d_{2}(v, \bar{v}) .
$$


The second term is bounded, and the first and the last can be estimated as in (4.1); thus, we obtain

$$
W_{2}(H) \leqslant C_{*}\left(E^{1 / 2}(u)+E^{1 / 2}(v)\right)+C,
$$

where $C$ equals $\operatorname{diam}(\mathrm{Harm})-2 C_{*} E_{*}^{1 / 2}$. Since, under the hypotheses of the theorem, the energy minimum $E_{*}$ is positive, this inequality can be re-written in the form (2.2).

Now we explain the proof of Theorem 2; it follows essentially the same idea. First, we discuss the version of Main Lemma I. By $d_{\infty}(u, v)$ we denote below the maximum of the distance function between maps $u$ and $v$.

Main Lemma II. Let $G$ be a finite graph and $(Y, d)$ be a locally compact Hadamard space. Let $\rho: \Gamma \rightarrow \operatorname{Isom}(Y)$ be a representation of the fundamental group $\Gamma=$ $\pi_{1}(G)$. Suppose that the moduli space Harm, formed by $\rho$-equivariant harmonic maps $T \rightarrow Y$, is non-empty and compact in $C^{0}$-topology. Then there exists a positive constant $C_{*}$ with the following property: for any continuous rectifiable $\rho$-equivariant map $u$ there exists a harmonic map $\bar{u} \in$ Harm such that

$$
d_{\infty}(u, \bar{u}) \leqslant C_{*}\left(L(u)-L_{*}\right),
$$

where $L_{*}=L(\bar{u})$ is the length minimum among $\rho$-equivariant maps.

Proof. First, without loss of generality we may assume that the maps $u: T \rightarrow Y$ under consideration are such that their restrictions to every edge are parameterised proportionally to the arc length. Second, as in the proof of Main Lemma I, it is sufficient to prove the lemma under the assumption that the distance $d_{\infty}(u, \bar{u})$ is not less than one.

Suppose the contrary. Then there exists a sequence of maps $u_{k}$ and harmonic maps $\bar{u}_{k}$ such that

$$
d_{\infty}\left(u_{k}, \bar{u}_{k}\right) \geqslant k\left(L\left(u_{k}\right)-L_{*}\right)
$$

we suppose that the $\bar{u}_{k}$ 's minimise the distance $\left\{d_{\infty}\left(u_{k}, v\right)\right.$, where $v \in$ Harm $\}$. Denote by $H_{s}^{k}$, where $s \in[0,1]$, a geodesic homotopy between $\bar{u}_{k}$ and $u_{k}$. Assuming that the parameter is proportional to the arc length and using the convexity of the length, relation (3.4), we obtain

$$
d_{\infty}\left(H_{s}^{k}\right) \geqslant k\left(L\left(H_{s}^{k}\right)-L\left(H_{0}^{k}\right)\right) .
$$

Choosing a sequence $s_{k} \in[0,1]$ such that the left-hand side above equals one, we conclude that $L\left(H_{s_{k}}^{k}\right)$ converges to $L_{*}$ as $k \rightarrow+\infty$. Since the lengths of $H_{s_{k}}^{k}$ are bounded and the edges of the $H_{s_{k}}^{k}$ 's are parameterised proportionally to the arc length, we see that the sequence of the $H_{s_{k}}^{k}$ 's is equicontinuous. Further, the compactness of Harm implies that the latter sequence is $d_{\infty}$-bounded. Now the Arzela-Ascoli 
theorem applies and there exists a subsequence converging in $d_{\infty}$-metric to a continuous map $\bar{v}$. The map $\bar{v}$ is clearly $\rho$-equivariant and length-minimising. Moreover, it has a constant-speed parameterisation and, hence, is harmonic. Now one gets a contradiction in the same way as in the proof of Main Lemma I.

Proof of Theorem 2. First, Lemma 1 carries over the case of $\rho$-equivariant maps of trees. In more detail, we need to start with a length minimising sequence which is uniformly Lipschitz continuous. The latter can be constructed by re-parameterising any length minimising sequence proportionally to the arc length on every edge. The rest of the proof (of Lemma 1) carries over without essential changes.

Now we simply follow the lines in the proof of Theorem 1 and use Main Lemma II instead of Main Lemma I.

We proceed with the proofs of Theorems 3 and 4. First, recall some notation. Let $\Lambda$ be a group acting properly and co-compactly by isometries on $Y$. For a homomorphism $\rho: \Gamma \rightarrow \Lambda$ by $Z$ we denote the centraliser of the image $\rho(\Gamma)$ in $\Lambda$. The group $Z$ acts naturally on the space of $\rho$-equivariant maps $u: \tilde{M} \rightarrow Y$ and, in particular, on the moduli space Harm.

Lemma 2. Under the hypotheses of Theorem 3, the moduli space Harm, formed by $\rho$-equivariant harmonic maps, is non-empty and the quotient Harm $/ Z$ is compact in $C^{0}$-topology.

Proof. We start with the existence of a $\rho$-equivariant harmonic map. By [11], Th. 2.6.4, there exists an energy minimising sequence $\left\{u_{i}\right\}$ of equivariant Lipschitz continuous maps, whose Lipschitz constants are uniformly bounded. Let $\Omega$ and $D$ be fundamental domains for the actions of $\Gamma$ on $\tilde{M}$ and $\Lambda$ on $Y$ respectively. Fix a point $x_{*} \in \Omega$. Then there exists a sequence of elements $h_{i} \in \Lambda$ such that the maps $h_{i} \cdot u_{i}$ send $x_{*}$ into the closure of $D$. Since the $h_{i}$ 's are isometries, the sequence $\left\{h_{i} \cdot u_{i}\right\}$ is also energy minimising and uniformly Lipschitz continuous. Moreover, since $\Lambda$ acts co-compactly, its fundamental domain $D$ is bounded, and the uniform Lipschitz continuity implies that the ranges $h_{i} \cdot u_{i}(\Omega)$ are contained in a bounded subset of $Y$. By the Arzela-Ascoli theorem there exists a subsequence, also denoted by $h_{i} \cdot u_{i}$, converging to a limit map $v$.

Now we define a homomorphism $\varphi: \Gamma \rightarrow \Lambda$ such that the limit map $v$ is $\varphi$ equivariant. For this fix a generator $g \in \Gamma$ and consider the points

$$
v(g \cdot x)=\lim \left(h_{i} \cdot u_{i}\right)(g \cdot x) \text { and } v(x)=\lim \left(h_{i} \cdot u_{i}\right)(x),
$$

where $x \in \Omega$. The triangle inequality implies that

$$
\left(h_{i} \rho(g) h_{i}^{-1}\right) \cdot v(x) \rightarrow v(g \cdot x) \quad \text { as } i \rightarrow+\infty .
$$

Now, since the action of $\Lambda$ is proper, the sequence $h_{i} \rho(g) h_{i}^{-1}$ contains a constant subsequence; we denote it value by $\varphi(g) \in \Lambda$. We use the $h_{i}$ 's of this subsequence 
for the same procedure for another generator in $\Gamma$. Repeating the process we define $\varphi$ on all generators. It then extends as a homomorphism $\varphi: \Gamma \rightarrow \Lambda$ and the map $v$ is $\varphi$-equivariant. As a result of this procedure, we also have a sequence $h_{i} \in \Lambda$ such that

$$
h_{i} \rho(g) h_{i}^{-1}=\varphi(g) \quad \text { for any } g \in \Gamma .
$$

This identity implies that the $h_{i}$ 's can be written in the form $k \cdot \overline{h_{i}}$, where $\overline{h_{i}} \in Z$, and the element $k \in \Lambda$ conjugates $\rho$ and $\varphi$. Now, since the sequence $h_{i} \cdot u_{i}$ converges to $v$, the sequence $\overline{h_{i}} \cdot u_{i}$ converges to $k^{-1} v$. Moreover, the latter is energy minimising and is formed by $\rho$-equivariant maps. Thus, the limit map $k^{-1} v$ is a harmonic $\rho$ equivariant map and the existence is demonstrated.

The compactness of Harm $/ Z$ follows by the same argument as above with the substitution of the sequence of harmonic maps for the energy minimising sequence $\left\{u_{i}\right\}$. By Proposition 3 the former sequence is also energy minimising, and by Proposition 4 is uniformly Lipschitz continuous; the argument above yields a sequence $\overline{h_{i}} \in Z$ such that $\overline{h_{i}} \cdot u_{i}$ converges to a $\rho$-equivariant harmonic map.

Proof of Theorem 3. Let $\mathscr{H}$ be a fundamental domain for the action of $Z$ on the moduli space Harm. First, Main Lemma I holds under a weaker hypothesis than the $L_{2}$-boundedness of Harm. More precisely, it is sufficient to assume that the domain $\mathscr{H}$ is bounded in the $L_{2}$-metric. Indeed, since the group $Z$ acts by isometries, one can suppose that the maps $\bar{u}_{k}$ 's (in the proof of Main Lemma I) belong to $\mathscr{H}$. The boundedness of the latter is then used to obtain the $W^{1,2}$-boundedness of the sequence $H_{s_{k}}^{k}$, relation (4.4). The rest of the proof stays unchanged.

Now the combination of Lemma 2 and estimate (4.1) yields the statement in the fashion similar to the proof of Theorem 1 .

Proof of Theorem 4. First, Main Lemma II holds under a weaker hypothesis than the compactness of the moduli space Harm. Similarly to the above, it is sufficient to assume that a fundamental domain for the action of $Z$ on Harm is compact. Further, Lemma 2 carries over the case of $\rho$-equivariant maps of trees; the proof follows essentially the same line of argument. The combination of this version of Lemma 2 with estimate (4.5) yields the statement in the same fashion as above.

\section{Finitely generated subgroups in isometry groups}

Recall that the action of a group $\Lambda$ on a metric space $(Y, d)$ by isometries defines an orbit pseudo-metric on $\Lambda$ :

$$
d_{y}(g, h)=d(g \cdot y, h \cdot y), \quad \text { where } g, h \in \Lambda,
$$

and $y \in Y$ is a fixed reference point. For another point $\bar{y} \in Y$ the pseudo-metrics $d_{y}$ and $d_{\bar{y}}$ are coarsely isometric; that is there exists a constant $C(=2 d(y, \bar{y}))$ such 
that

$$
d_{\bar{y}}(g, h)-C \leqslant d_{y}(g, h) \leqslant d_{\bar{y}}(g, h)+C .
$$

First, we show that the $L_{\infty}$-width inequalities imply an estimate for the conjugating element in the orbit pseudo-metric.

Lemma 3. Let $\boldsymbol{G}$ be a semi-simple Lie group all of whose simple factors are noncompact. Let $\Lambda$ be an irreducible lattice in $\boldsymbol{G}$ and $\left(a_{i}\right)_{1 \leqslant i \leqslant N}$ be a finite list of elements in $\Lambda$ which does not fix a point on the ideal boundary of the associated symmetric space. Then for any conjugate (in $\Lambda$ ) list $\left(b_{i}\right)_{1 \leqslant i \leqslant N}$ any conjugating element $g \in \Lambda$, $b_{i}=g^{-1} a_{i} g$ satisfies the inequality

$$
d_{y}(g, e) \leqslant C_{*} \sum_{i=1}^{N}\left(d_{y}\left(a_{i}, e\right)+d_{y}\left(b_{i}, e\right)\right),
$$

where $y \in Y$ is a reference point, and the constant depends only on the conjugacy class of the list $\left(a_{i}\right)$.

Proof. Let $Y$ be a symmetric space associated with the Lie group $\boldsymbol{G}$. Under the hypotheses on $\boldsymbol{G}$, the natural $\boldsymbol{G}$-invariant Riemannian metric on $Y$ defines a distance $d$ which makes $Y$ into an Hadamard space.

Consider a bouqet of $N$ copies of a circle; denote by $\Gamma=*_{i=1}^{N} \mathbf{Z}$ its fundamental group and by $T$ its universal cover. Define a homomorphism $\rho: \Gamma \rightarrow \Lambda$ by the rule: the generator of the $i$ th copy of $\mathbf{Z}$ maps into $a_{i}$. For a fixed reference point $y \in Y$ consider the graph in $Y$ whose vertices are points $g \cdot y$, where $g$ is a word in the alphabet $\left(a_{i}\right)$. The edges are geodesic arcs; two points $g_{1} \cdot y$ and $g_{2} \cdot y$ are joined by an edge if and only if $g_{1}^{-1} g_{2}$ is an element $a_{i}$ or its inverse. Suppose that each edge is parameterised proportionally to the arc length. Such a parameterisation defines a $\rho$-equivariant map $u: T \rightarrow Y$, whose length $L(u)$ is given by the sum $\sum_{i=1}^{N} d\left(a_{i} y, y\right)$.

Analogously, for a conjugate list $\left(b_{i}\right)_{1 \leqslant i \leqslant N}$ one defines a $\left(g^{-1} \rho g\right)$-equivariant map $v: T \rightarrow Y$, where $g$ is a conjugating element. Note that the map $g \cdot v$ is $\rho$ equivariant and its length $L(g \cdot v)$ coincides with $L(v)=\sum_{i=1}^{N} d\left(b_{i} y, y\right)$. By the hypotheses of the lemma, Theorem 2 applies and we have

$$
d(g \cdot y, y) \leqslant W_{\infty}(H) \leqslant C_{*}(L(u)+L(v)),
$$

where $H$ is a homotopy between $u$ and $g \cdot v$. Now the combination with the expressions for the lengths finishes the proof.

Proof of Theorem 5. The statement is a direct consequence of Lemma 3 and the solution of Kazhdan's conjecture in [12]. The latter says that the word metric (with respect to some finite set of generators) on an irreducible lattice $\Lambda$ is quasi-isometric to the orbit metric (with respect to the action on the associated symmetric space or Euclidean building) provided $\boldsymbol{G}$ is semi-simple and its rank $\geqslant 2$. 
Lemma 4. Let $Y$ be a locally compact Hadamard space and $\Lambda$ be a group acting properly and co-compactly by isometries on $Y$. Then for any finite conjugate lists $\left(a_{i}\right)_{1 \leqslant i \leqslant N}$ and $\left(b_{i}\right)_{1 \leqslant i \leqslant N}$ of elements in $\Lambda$ there exists an element $g \in \Lambda$ with $b_{i}=g^{-1} a_{i} g$ such that

$$
d_{y}(g, e) \leqslant C_{*} \sum_{i=1}^{N}\left(d_{y}\left(a_{i}, e\right)+d_{y}\left(b_{i}, e\right)\right)+C,
$$

where $y \in Y$ is a reference point, and the constants depend only on the conjugacy class of the list $\left(a_{i}\right)$.

Proof. The proof follows the same line of argument as the proof of Lemma 3 with the use of Theorem 4 instead of Theorem 2.

Proof of Theorem 6. By the Švarc-Milnor lemma [1], I.8.19, the word and orbit metrics on $\Lambda$ are quasi-isometric. The combination of this with Lemma 4 implies the first statement of the theorem. Further, by [1], III.Г.1.4, the word problem in $\Lambda$ is soluble. This yields the algorithm deciding the conjugacy of finite lists in the following fashion. If there exists an element conjugating two given lists, then it belongs to the finite subset of $\Lambda$ formed by elements satisfying the bound (2.3). Using the solubility of the word problem, the algorithm checks all elements from this finite set.

\section{References}

[1] M. R. Bridson and A. Haefliger, Metric spaces of non-positive curvature. Grundlehren Math. Wiss. 319, Springer-Verlag, Berlin 1999. Zbl 0988.53001 MR 1744486

[2] M. R. Bridson and J. Howie, Conjugacy of finite subsets in hyperbolic groups. Internat. J. Algebra Comput. 15 (2005), 725-756. Zbl 1083.20032 MR 2160576

[3] Bridson, M. Problems concerning hyperbolic and CAT(0)-groups. Preprint, 2007.

[4] P. Eberlein and B. O’Neill, Visibility manifolds. Pacific J. Math. 46 (1973), 45-109. Zbl 0264.53026 MR 0336648

[5] J. Eells and B. Fuglede, Harmonic maps between Riemannian polyhedra. Cambridge Tracts in Math. 142, Cambridge University Press, Cambridge 2001. Zbl 0979.31001 MR 1848068

[6] F. Grunewald and D. Segal, Some general algorithms. I: Arithmetic groups. Ann. of Math. (2) 112 (1980), 531-583. Zbl 0457.20047 MR 595206

[7] T. Kappeler, S. Kuksin, and V. Schroeder, Perturbations of the harmonic map equation. Commun. Contemp. Math. 5 (2003), 629-669. Zbl 1063.58012 MR 2003212

[8] T. Kappeler, V. Schroeder, and S. Kuksin, Poincaré inequalities for maps with target manifold of negative curvature. Moscow Math. J. 5 (2005), 399-414, 494. Zbl 1093.53037 MR 2200758 
[9] G. Yu. Kokarev, The property of compactness of the quasi-linearly perturbed harmonicmap equation. Mat. Sb. 194 (2003), 105-118; English transl. Sb. Math. 194 (2003), 1055-1068. Zbl 1071.58014 MR 2020380

[10] G. Kokarev, A note on Morse inequalities for harmonic maps with potential and their applications. Ann. Global Anal. Geom. 33 (2008), 101-113. Zbl 1144.53081 MR 2379939

[11] N. J. Korevaar and R. M. Schoen, Sobolev spaces and harmonic maps for metric space targets. Comm. Anal. Geom. 1 (1993), 561-659. Zbl 0862.58004 MR 1266480

[12] A. Lubotzky, S. Mozes, and M. S. Raghunathan, The word and Riemannian metrics on lattices of semisimple groups. Inst. Hautes Études Sci. Publ. Math. 91 (2000), 5-53. Zbl 0988.22007 MR 1828742

[13] H. A. Masur and Y. N. Minsky, Geometry of the complex of curves. II: Hierarchical structure. Geom. Funct. Anal. 10 (2000), 902-974. Zbl 0972.32011 MR 1791145

[14] Yu. G. Reshetnyak, Inextensible mappings in a space of curvature no greater than $K$. Sibirsk. Mat. Ž. 9 (1968), 918-927; English transl. Siberian Math. J. 9 (1968), 683-689. Zbl 0167.50803 MR 0244922

[15] T. Riley, The geometry of the conjugacy problem. Talk given at Fields Workshop on Asymptotic Group Theory and Cryptography, Ottawa, December 15, 2007; http://www.math.cornell.edu/ riley/

Received January 30, 2010; revised January 22, 2012

G. Kokarev, Mathematisches Institut, Ludwig-Maximilians-Universität München, Theresienstr. 39, 80333 München, Germany

E-mail: Gerasim.Kokarev@mathematik.uni-muenchen.de 